\title{
NANOINDENTACIONES Y ULTRAESTRUCTURA EN MADERA DE EUCALYPTUS NITENS CON MICRO Y MESO GRIETAS
}

\section{NANOINDENTATIONS AND ULTRASTRUCTURE IN EUCALYPTUS NITENS WITH MICRO AND MESOCRACKS}

\author{
William Gacitua E ${ }^{1}$, Aldo Ballerini A ${ }^{1}$, Jean Pierre Lasserre ${ }^{2}$, David Bahr ${ }^{3}$
}

\begin{abstract}
RESUMEN
Se desarrolló una metodología basada en nanoindentaciones y análisis de la ultraestructura de células de madera de Eucalyptus nitens (Deane et Maiden) para explorar los factores que generan la aparición de fallas en la madera debido a tensiones de crecimiento. Se estudió madera normal y madera con mesogrietas. Existieron diferencias significativas en la frecuencia de vasos y módulo de elasticidad de la pared S2 entre los dos tipos de madera estudiadas. Se identificó como causa de la fractura de la lámina media y pared S1, fractura definida como mesogrieta, el factor de concentración de esfuerzos y la capacidad de almacenamiento de energía por parte de las fibras de madera, presentando ambos factores diferencias significativas entre las muestras de madera analizadas.
\end{abstract}

Palabras claves: nano-mecánica, pared celular, colapso, grietas, tensiones de crecimiento.

\begin{abstract}
Using nanoindentations and ultrastructure analysis, we developed a methodology to explore factors that may contribute to failures in Eucalyptus nitens (Deane et Maiden) due to growth stresses. Two types of wood were analyzed; normal wood and wood with meso-cracks. Factors contributing to cracking included the vessel frequency and mechanical properties of the S2 layer measured with nanoindentations. Micro and mesocracks propagated throughout the middle lamella and S1 layer due to an increased stress concentration that was mainly caused by vessels and the characteristic strain energy density of the cell wall.
\end{abstract}

Keywords: nano-mechanics, cell wall, collapse, cracking, growth stresses.

\section{INTRODUCCION}

Durante el crecimiento del árbol, esfuerzos o tensiones internas se pueden desarrollar en su estructura. Estas tensiones son usualmente llamadas tensiones de crecimiento (Smith et al. 2003). Estas tensiones se desarrollan en el cambium a medida que este agrega una capa nueva de madera al tronco. Hoy en día no existe consenso sobre la causa exacta de estas tensiones de crecimiento y como estas son generadas. Existen dos hipótesis que usualmente se discuten en la comunidad científica; la hipótesis del hinchamiento de la lignina y la hipótesis de la tensión en la celulosa (Bamber, 1978). La primera hipótesis se refiere al depósito o inserción de lignina entre microfibrillas de celulosa causando expansión

${ }^{1}$ Depto. Ingeniería en Maderas, Universidad del Bio-Bio, Concepción. Chile. aballeri@ubiobio.cl 
transversal de las células de madera. En el caso de la celulosa en tensión, esfuerzos de tracción se generan a partir de la contracción de la celulosa durante su cristalización. Tensiones de crecimiento consisten en tracción longitudinal en las capas exteriores del tronco del árbol y esfuerzos de compresión en el centro de este. El tronco del árbol esta sometido tensiones o esfuerzos de tracción en la dirección radial, mientras que en la dirección tangencial, las tensiones de crecimiento cambian desde compresión en la periferia a tracción cerca de la médula (Alhasani, 1999).

El objetivo de esta investigación fue explorar y estudiar el efecto de la anatomía de las células de madera de Eucalyptus nitens y sus propiedades mecánicas a nanoescala en el inicio y propagación de micro y mesogrietas debido a tensiones de crecimiento.

\section{METODOLOGIA}

Se utilizaron muestras de Eucalyptus nitens de árboles de 16 años provenientes de ensayos de progenie de polinización abierta. Estos se realizaron en Rucamanqui 2 sector 4110 predio El Lavado, ubicados a $14 \mathrm{~km}$ al Noreste de la Comuna de Yungay, VIII Región. El suelo corresponde a uno del tipo trumao. De estos ejemplares se cortaron rodelas de $5 \mathrm{~cm}$ de espesor a $1.3 \mathrm{~m}$ desde la base del árbol. Las rodelas fueron acondicionadas por 5 meses bajo sombra y a temperatura ambiente hasta alcanzar la condición seca. Del total de rodelas disponibles, cuatro de ellas fueron seleccionadas de acuerdo a la presencia o ausencia visible de grietas. La tabla 1 muestra las principales características de las muestras analizadas. Se hace la diferencia en el tipo de falla o fractura de la madera, entonces se define: Mesogrieta como una falla visible en la estructura del sólido madera y que se ubicó exclusivamente en madera de primavera. Las microgrietas son fallas no visibles por el ojo humano y solo detectables usando SEM (Scanning Electron Microscopy).

Tabla 1: Características de muestras secas de Eucalyptus nitens.

\begin{tabular}{cccc}
\hline $\begin{array}{c}\text { Árbol } \\
\text { número }\end{array}$ & Familia & $\begin{array}{c}\text { Densidad básica } \\
\mathrm{kg} / \mathrm{m}^{3}\end{array}$ & $\begin{array}{c}\text { Estado de madera de } \\
\text { primavera }\end{array}$ \\
\hline 621 & 2154 & 493 & Madera normal \\
646 & 2155 & 469 & $\begin{array}{c}\text { Madera normal } \\
\text { Presencia de } \\
\text { microgrietas }\end{array}$ \\
\hline 663 & 2157 & 487 & $\begin{array}{c}\text { Mesogrietas en anillo } \\
\text { de primavera }\end{array}$ \\
714 & 2157 & 487 & $\begin{array}{c}\text { Mesogrietas en anillo } \\
\text { de primavera }\end{array}$ \\
\hline
\end{tabular}


Para el análisis de elementos anatómicos de la madera de primavera de Eucalyptus nitens se utilizó un microscopio electrónico de barrido TSL SEM Tex Laboratories, Inc. con $15 \mathrm{kV}$ y 20 a $25 \mathrm{~mm}$ de distancia de trabajo. Posteriormente se utilizó el software Image-pro plus para tratamiento de imágenes y mediciones a microescala de la estructura celular de la madera, particularmente la madera de primavera que es donde el material celular presentó grietas. En cada árbol, se cortó dos muestras cúbicas de $3 \mathrm{~mm}$ que fueron posteriormente recubiertas con oro para observación en el SEM. Para la cuantificación de medidas y parámetros de la estructura anatómica del Eucalyptus nitens, se utilizaron áreas de interés (AOI) de $2000 \times 2500 \mu \mathrm{m}^{2}$; dos AOI por cada cubo fueron evaluadas. Otro grupo de pequeñas muestras de similares dimensiones y ubicación, fueron cortadas en una de sus secciones transversales usando un cuchillo de diamante montado en un ultramicrótomo. De esta manera se obtuvo secciones transversales destinadas al ensayo de nanoindentación (dos cubos por árbol). En el caso de muestras con mesogrietas, usadas para la observación con SEM y nanoindentación, los cubos fueron cortados desde una zona adyacente a la cara de la falla o cara de la mesogrieta (ver fig. 1b y fig. 6b, 6c). El ensayo de nanoindentación de paredes celulares de pequeñas muestras se realizó en un nanoindentador Triboscope Hysitron Nanomechanical test Instrument equipado con un transductor de fuerza. Las indentaciones fueron realizadas con un indentador tipo pirámide triangular Berkovich y empleando un ciclo de carga con fuerza nominal máxima de $300 \mu \mathrm{N}$. Para la determinación del módulo de elasticidad y esfuerzo de fluencia, se indentaron 10 fibras por cubo. En un ensayo de nanoindentación, la carga máxima, la penetración del indentador y la rigidez inicial (pendiente de la curva de descarga), fueron obtenidas. Conociendo la geometría del indentador se puede determinar el área de contacto entre el indentador y el material. Luego, el módulo reducido $\left(E_{r}\right)$ se determina de acuerdo a la siguiente ecuación (Gindl et al., 2004):

$$
E_{r}=\left[\frac{1-v_{s}^{2}}{E_{s}}+\frac{1-v_{\mathrm{i}}^{2}}{\mathrm{E}_{\mathrm{i}}}\right]^{-1}
$$

donde, los subíndices $s$ y $i$ representan la muestra (capa S2 de la pared celular) e indentador respectivamente. $v$ es la razón de Poisson; el módulo del indentador $E_{i}$ es constante e igual a $1240 \mathrm{GPa}$ con una razón de Poisson de 0.07. Una razón de Poisson igual a 0.35 fue considerada para el Eucalyptus nitens (Gibson y Ashby, 1997). Finalmente se determina el módulo de elasticidad o módulo de Young de la muestra $\left(E_{s}\right)$.

\section{RESULTADOS Y DISCUSIÓN}

Una primera diferencia en términos de comportamiento mecánico de las muestras se percibió durante la preparación y corte de los cubos de madera a montar en el ultramicrótomo. Al cortar las muestras 621 y 646 (muestras sin mesogrietas) usando una hoja de ultrafilo, la resistencia al corte perpendicular a la fibra fue menor a la detectada para las muestras 663 y 714 (muestras con mesogrietas). Esto pareció un indicador de resistencia ofrecida por la madera y que podría explicarse por las propiedades a nanoescala de las estructuras de las células de Eucalyptus nitens (pared celular y lámina media). Al parecer las estructuras celulares de las muestras no colapsadas permite una mayor deformación plástica sin ruptura de la estructura del Eucalyptus nitens (grietas), generando así una baja resistencia al corte. La mayor resistencia al corte perpendicular a las fibras de las muestras 663 y 714 es además explicada por la presencia de células colapsadas (fig. 6).

\section{Análisis SEM y micromecánica de la estructura del Eucalyptus nitens.}

En general, los vasos no presentan deformación plástica permanente significativa; esto debido a su mayor espesor de pared (fig. 4b, árbol 621); El efecto de concentración de esfuerzos alrededor de un pequeño orificio (vaso) en el sólido madera es más evidente en muestras con mesogrietas. Por otro 
lado, las microgrietas se inician en los vasos y en una zona donde teóricamente se encuentra el mayor esfuerzo resultante del efecto "concentración de esfuerzo" provocado por un vaso (fig. 4a y fig. 5b, 5c, 5d). Esta microgrieta se propaga sin mayor resistencia por las zonas "débiles" de la estructura de la madera, que corresponden a la lámina media y pared S1. Eventualmente esta microgrieta se trasformaría en mesogrieta dependiendo del nivel de tensiones y de cómo estas se distribuyen y disipan en la estructura anatómica del Eucalyptus nitens (fig. 6d).

Es evidente que la presencia de vasos y sus características geométricas, frecuencia y propiedades mecánicas juegan un rol fundamental en el agrietamiento de la madera. Primero, en términos de dimensiones, no existen diferencias significativas en el diámetro de vasos medido en las cuatro muestras o árboles (fig . 2 y tabla 2).

Por otro lado y de acuerdo a ecuaciones clásicas de la mecánica, si la frecuencia de estas imperfecciones (orificios) en un sólido es mayor, el factor de concentración de esfuerzos también crece. En términos referidos a la madera, a mayor concentración de vasos mayor es la concentración de esfuerzos y probabilidad de aparición de microgrietas y posteriormente fallas (mesogrietas y rajaduras).

Dado que el área de espacios vacíos en la sección transversal de un sólido, en este caso madera de Eucalyptus nitens, esta directamente relacionado con el factor de concentración de esfuerzos, se define el parámetro área vasos por área de interés expresado en $\mu \mathrm{m}^{2} / \mathrm{mm}^{2}$ (tabla 2). Este parámetro fue significativamente mayor en muestras con mesogrietas visibles (tabla 2 y fig. 1). El otro parámetro relacionado con la anatomía de la madera fue definido como número vasos por $\mathrm{mm}^{2}$. Se observó que ambos parámetros aquí definidos se correlacionan muy bien con la respuesta (microgrietas y mesogrietas) observada como falla en el material (fig. 3). A mayor valor de estos dos parámetros, mayor es la probabilidad de aparición de una falla irrecuperable. En este escenario se requiere, en investigaciones futuras, definir el valor crítico de estos parámetros del material que puedan resultar en defectos y fallas producto de las tensiones de crecimiento del Eucalyptus nitens y su transformación en productos.

Adicionalmente, hay diferencias y solamente observadas a microescala entre muestras sin mesogrietas visibles. Esta diferencia pudo ser cuantificada gracias a los parámetros Número de vasos $/ \mathrm{mm}^{2}$ y área vasos/AOI. La muestra 621, con menor número de vasos $/ \mathrm{mm}^{2}$ y menor área de vasos/AOI, no presentó microgrietas (tabla 2, fig. 3 y fig. 4b). Sin embargo, se detecto con SEM el inicio de microgrietas particularmente en muestra 646, la que también presenta valores reducidos de los parámetros mencionados.

Table 2: Características anatómicas de muestras de Eucalyptus nitens.

\begin{tabular}{|c|c|c|c|}
\hline Muestra & $\begin{array}{c}\text { Diámetro promedio } \\
\text { Vaso } \\
\mu \mathrm{m}\end{array}$ & $\begin{array}{c}\text { Número } \\
\text { vasos } / \mathrm{mm}^{2}\end{array}$ & $\begin{array}{c}\text { Área vasos/AOI } \\
\mu \mathrm{m}^{2} / \mathrm{mm}^{2}\end{array}$ \\
\hline 621 & 130.7 & 4.45 & 1614 \\
\hline 646 & 132.1 & 5.92 & 1805 \\
\hline 663 & 125.6 & 7.88 & 2571 \\
\hline 714 & 134.6 & 6.36 & 2384 \\
\hline
\end{tabular}




\section{Analogía entre mecánica de sólidos y estructura celular del Eucalyptus nitens.}

La figura 7 muestra el perfil de esfuerzos tangenciales a un orificio en un sólido cualquiera sometido a tensiones o esfuerzos. En términos de estructura de la madera, esta imperfección u orificio esta representada por la presencia de vasos que interrumpen la estructura celular del Eucalyptus nitens. Las tensiones internas en madera de Eucalyptus nitens (de crecimiento o producto de contracciones por pérdida de humedad) actúan en todas direcciones, pero son mayores en la dirección tangencial a los anillos de crecimiento. En este orificio o vaso, el esfuerzo máximo (positivo o tensión) se proyecta desde el centro geométrico del vaso y perpendicular a la dirección del esfuerzo aplicado tangencialmente al anillo de crecimiento resultando así, el inicio y propagación de la fractura de la madera de primavera en la dirección radial, la cual coincide con la dirección del mayor esfuerzo concentrado alrededor del vaso. Esfuerzos combinados de tracción y cizalle podrían explicar entonces las falla observada con SEM. De acuerdo a la mecánica de sólidos (Sanford, 2003; Graig, 2000; Ugural y Fenster, 1995), el esfuerzo tangencial máximo generaría el efecto cuña en el sólido o estructura celular de la madera, iniciando la microgrieta y posteriormente la mesogrieta a partir del centro geométrico del vaso (ver figuras $4 b, 5 b, 5$ c y $6 b$ ). Los esfuerzos concentrados se liberan inicialmente en la microgrieta la que se transforma en una falla mayor o mesogrieta dependiendo del nivel de esfuerzos concentrados en la periferia del orificio o vaso; la falla se propaga finalmente a través de las estructuras poliméricas mas débiles de la madera, las que están localizadas en la lamina media formada básicamente por lignina (fig. 5d y fig. 6b, 6c).

Para el modelo de un sólido interrumpido en su volumen por un orificio, el factor de concentración de esfuerzos (razón entre máximo esfuerzo en la periferia del orificio y el esfuerzo aplicado) es igual a 3. El factor de concentración de esfuerzos es independiente del diámetro del orificio (Sanford, 2003).

Las razones de la variación del diámetro del vaso, su espesor y distribución en la estructura celular del Eucalyptus nitens tienen varias probables razones, las cuales no fueron consideradas en esta investigación.

No se encontraron referencias para Eucalyptus nitens en términos de variabilidad en características de los vasos y/o características anatómicas. Sin embargo se puede mencionar que existen referencias de este tipo pero para otras especies. Leitch (2001) estableció que el número de vasos por área en Eucalyptus globulus, aumenta desde la base del árbol hasta su copa. Desde médula a corteza, el área media del vaso aumenta mientras que la frecuencia de estos disminuye en la misma dirección (Leal et al. 2003).

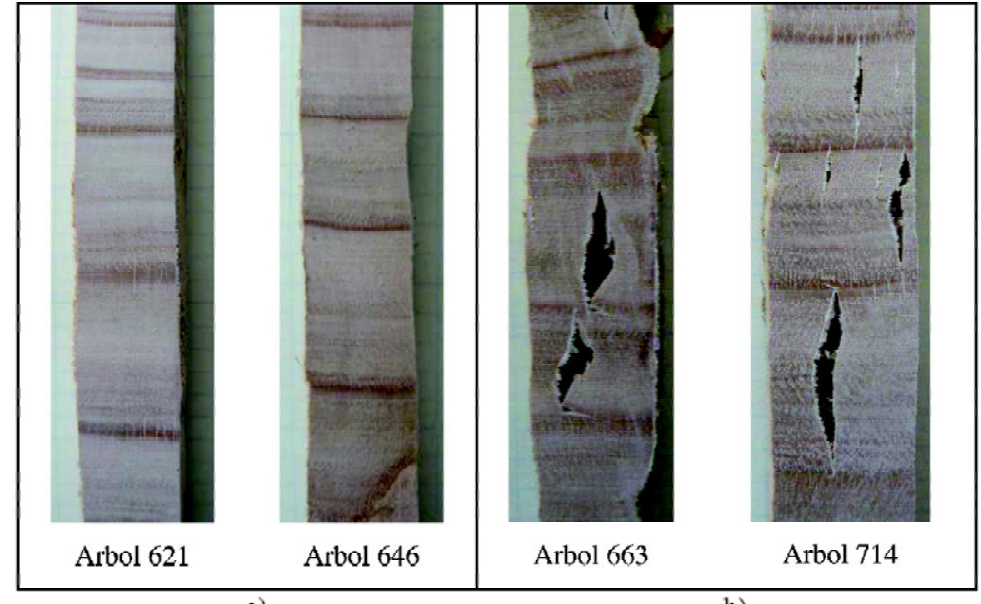

a)

b)

Figura 1: a) Muestras de madera normal de Eucalyptus nitens (familia 2154 y 2155) y, b) Muestras de madera agrietada en anillo de primavera (familia 2157). 


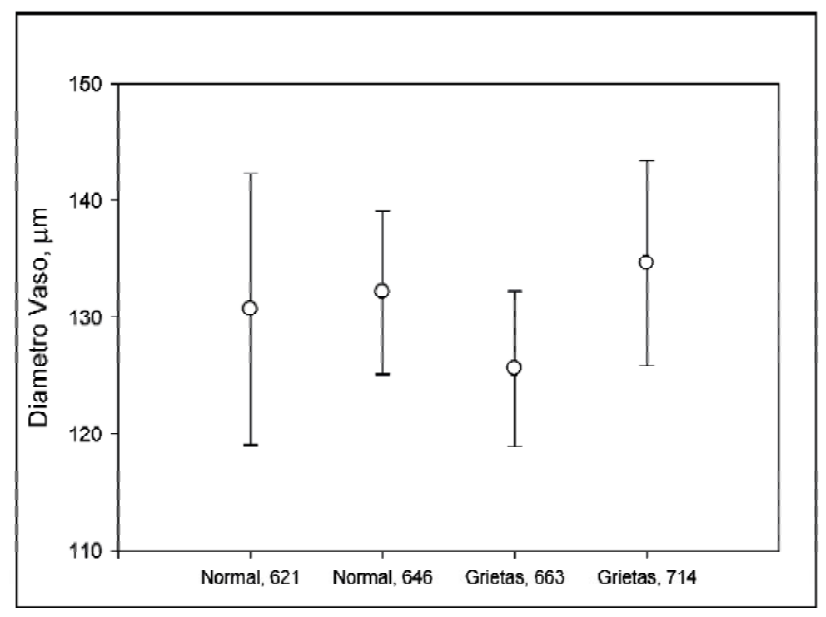

Figura 2: Variabilidad del diámetro de vasos localizados en madera de primavera de Eucalyptus nitens. Intervalo de confianza de la media, $95 \%$ de confianza.

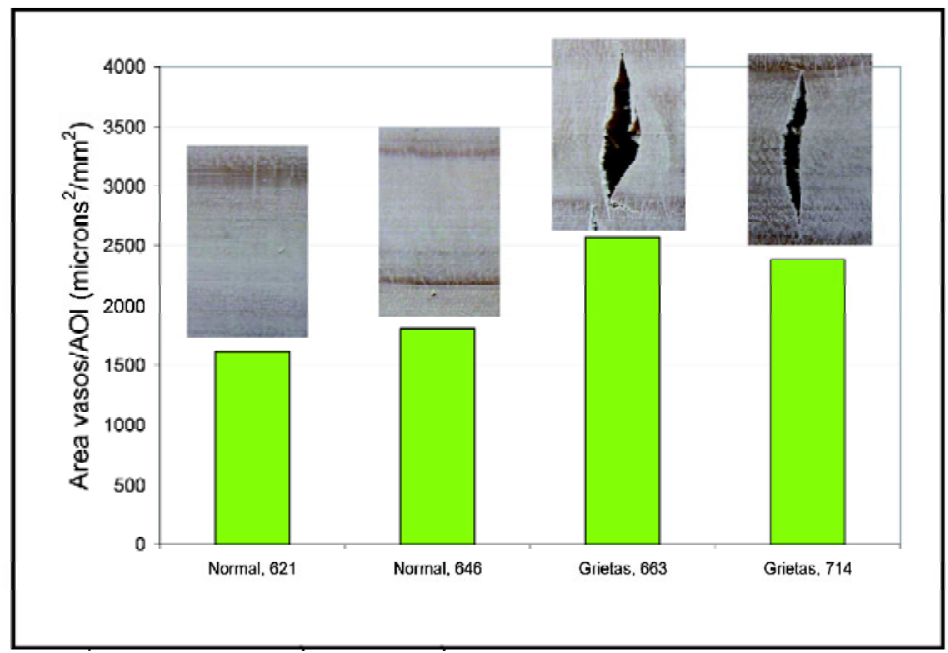

Figura 3: Área de vasos $\left(\mu \mathrm{m}^{2}\right)$ en un $\mathrm{mm}^{2}$ de madera de primavera de Eucalyptus nitens; madera normal y madera con mesogrietas.

\section{Nanoindentaciones en la pared celular.}

La figura 8 muestra la marca de una nanoindentación sobre la capa S2 de la pared celular del Eucalyptus nitens. Valores de módulo de elasticidad y esfuerzo de fluencia de esta capa para las muestras aquí analizadas son presentados en la figura 9. El módulo de elasticidad de la capa S2 de muestras con mesogrietas es significativamente mayor al de madera de Eucalyptus nitens denominada madera normal. Por otro lado, el esfuerzo de fluencia no presentó diferencias significativas entre madera normal y madera con mesogrietas. La nanocaracterización permitió establecer diferencias entre los dos tipos de madera de Eucalyptus nitens aquí analizados que combinada con la cuantificación de parámetros de la anatomía de la madera, explican el tipo e falla observada en la madera debido a tensiones de crecimiento y tensiones producto de la pérdida de humedad del material. En términos del efecto de la anatomía del Eucalyptus nitens, la mecánica de sólidos ayuda a explicar y analizar 
teóricamente el efecto de concentración de esfuerzos entre las muestras analizadas, siendo el tamaño del vaso y su frecuencia en el sólido madera, de significancia.

Por otro lado, el uso de nanoindentaciones permitió explorar en aspectos desconocidos de la micromecánica de la pared celular que influyen en como se disipan las tensiones en la estructura del Eucalyptus nitens. Asumiendo que no hay cambios en el esfuerzo de fluencia entre madera normal y madera con mesogrietas (figura 9b), presentando estas solo diferencias significativas en el módulo de elasticidad, se establece que la energía de deformación (área bajo la curva esfuerzo vs. deformación) que puede absorber la madera normal es mayor, permitiéndole así soportar mayores esfuerzos sin sufrir deformaciones permanentes y significativas en su estructura celular (Ugural y Fenster, 1995).

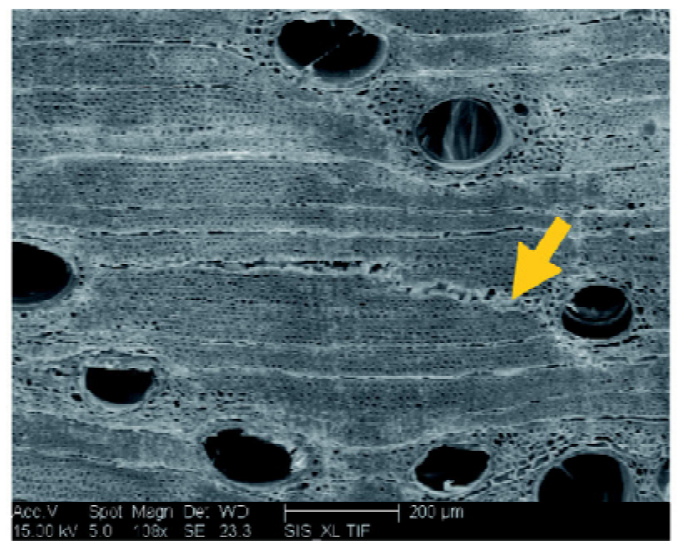

a)

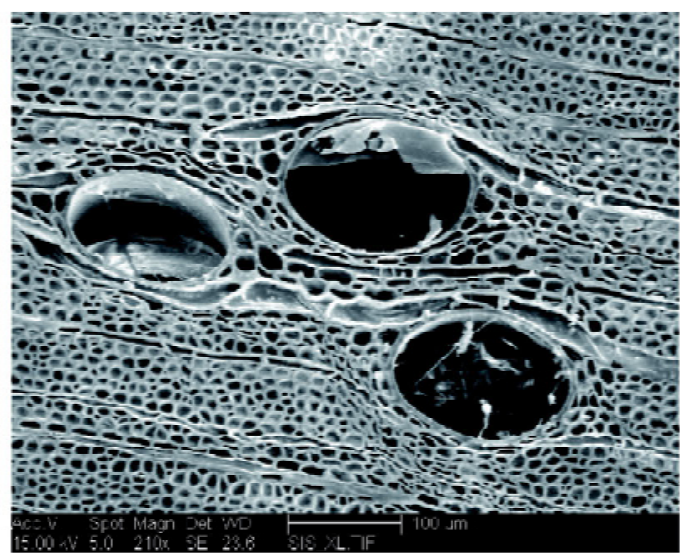

b)

Figura 4: Imágenes SEM de muestra desde árbol 646; se aprecia colapso alrededor de vasos que se encuentran en mayor frecuencia que en muestra 621, además de microgrieta que conecta a dos vasos (flecha) (a). Sección transversal de muestra desde árbol 621; aparece leve colapso celular alrededor de vasos (b). 


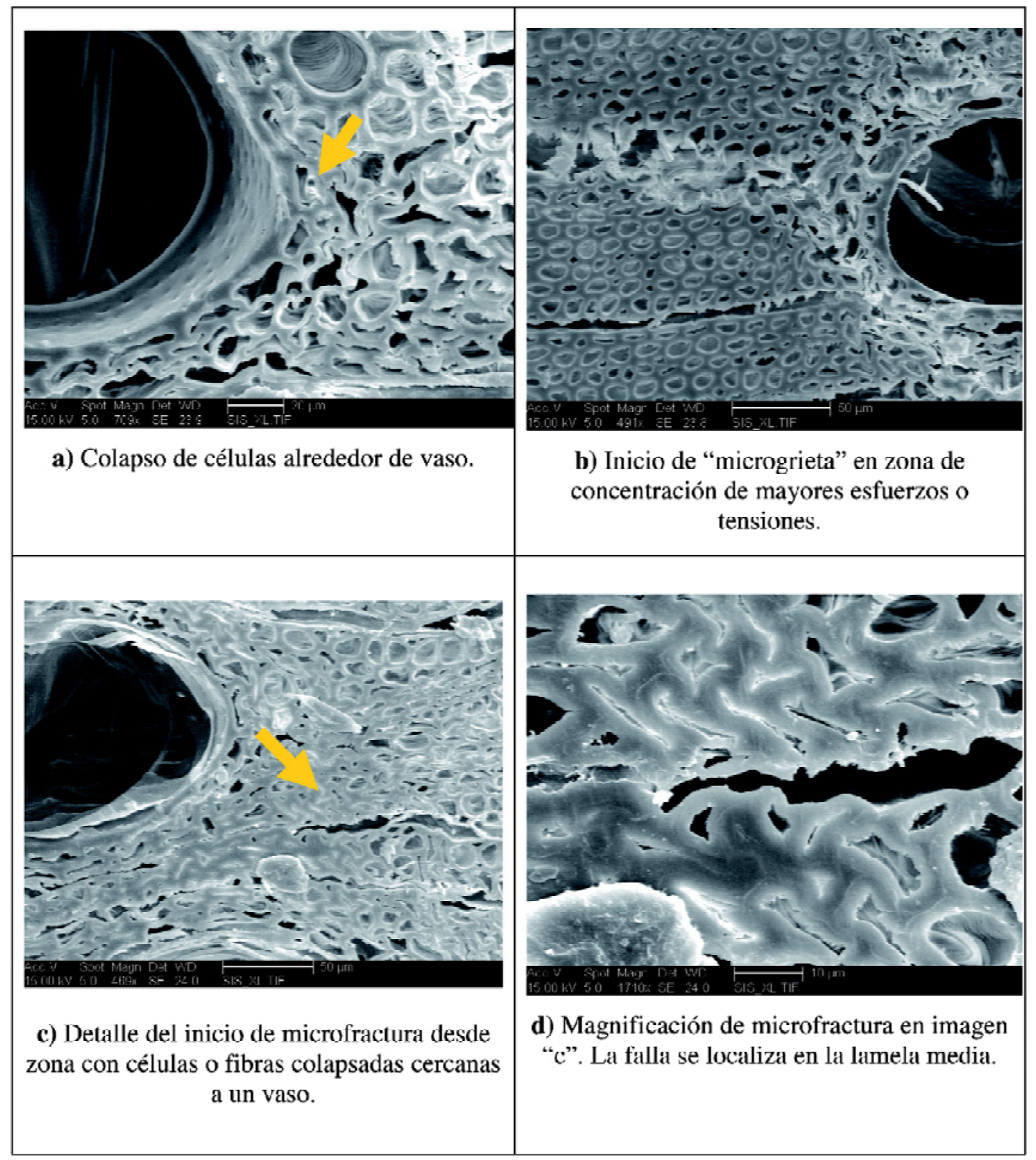

Figura 5: Imágenes SEM del colapso e inicio de microgrietas en "madera normal" de Eucalyptus nitens (muestra desde árbol 646). 


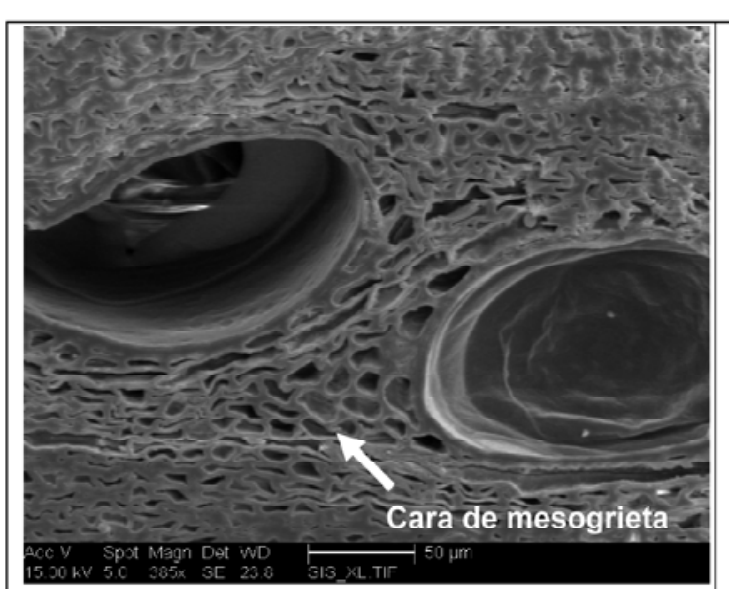

a)

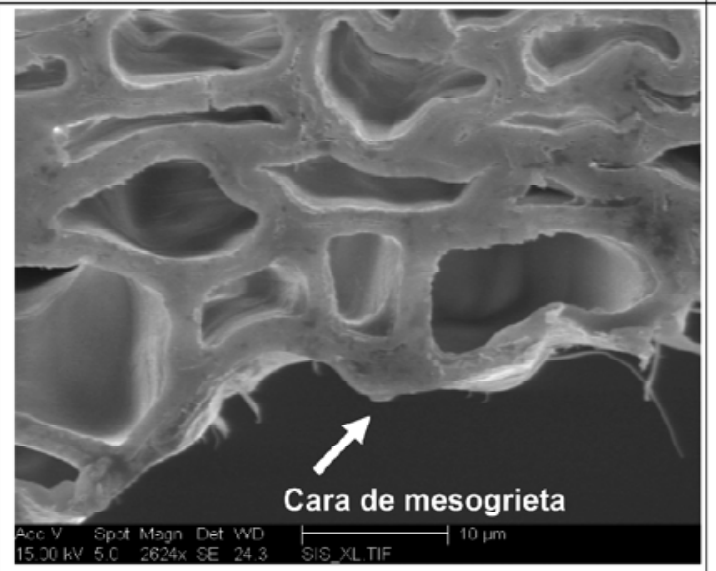

c)

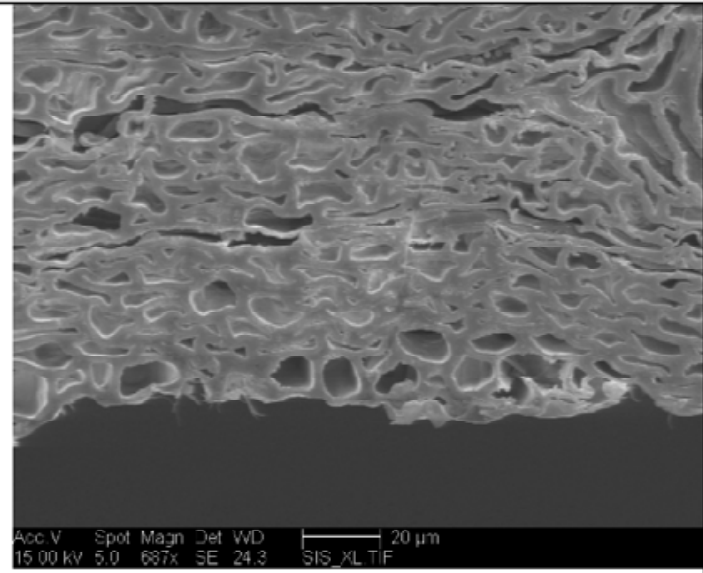

b)

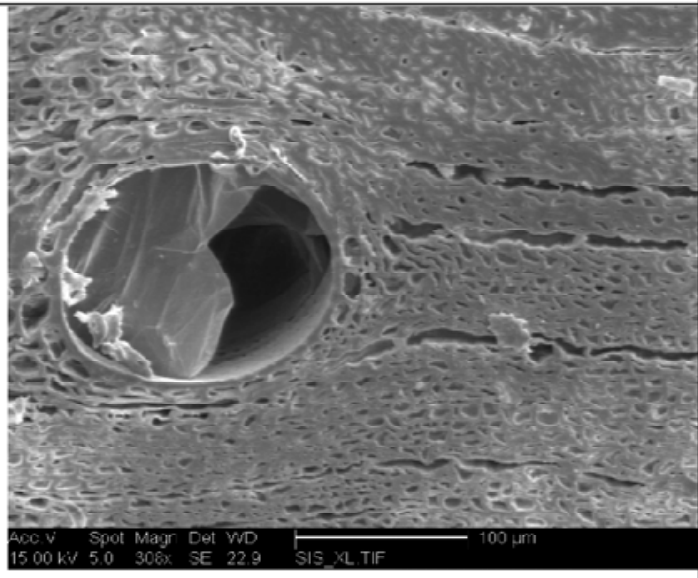

d)

Figura 6: Imágenes SEM de la deformación plástica critica de células alrededor de vasos producto de concentración de tensiones en árbol 663 (a). Cara de mesogrieta en muestra de árbol 663 (b). Evidencia de falla en lamina media, árbol 663 (c). Inicio de microfallas alrededor del vaso en muestra desde árbol 714 (d). 


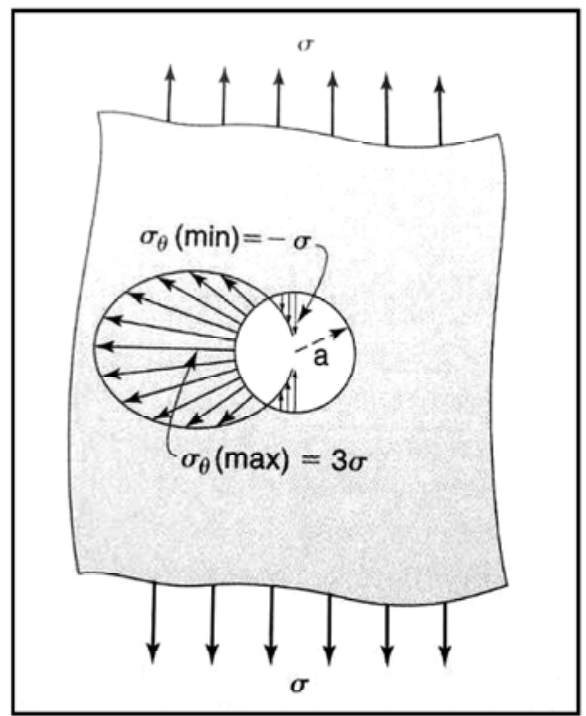

Figura 7: Distribución de esfuerzos tangenciales alrededor de orificio en un sólido imperfecto (Sanford R.J., 2003).

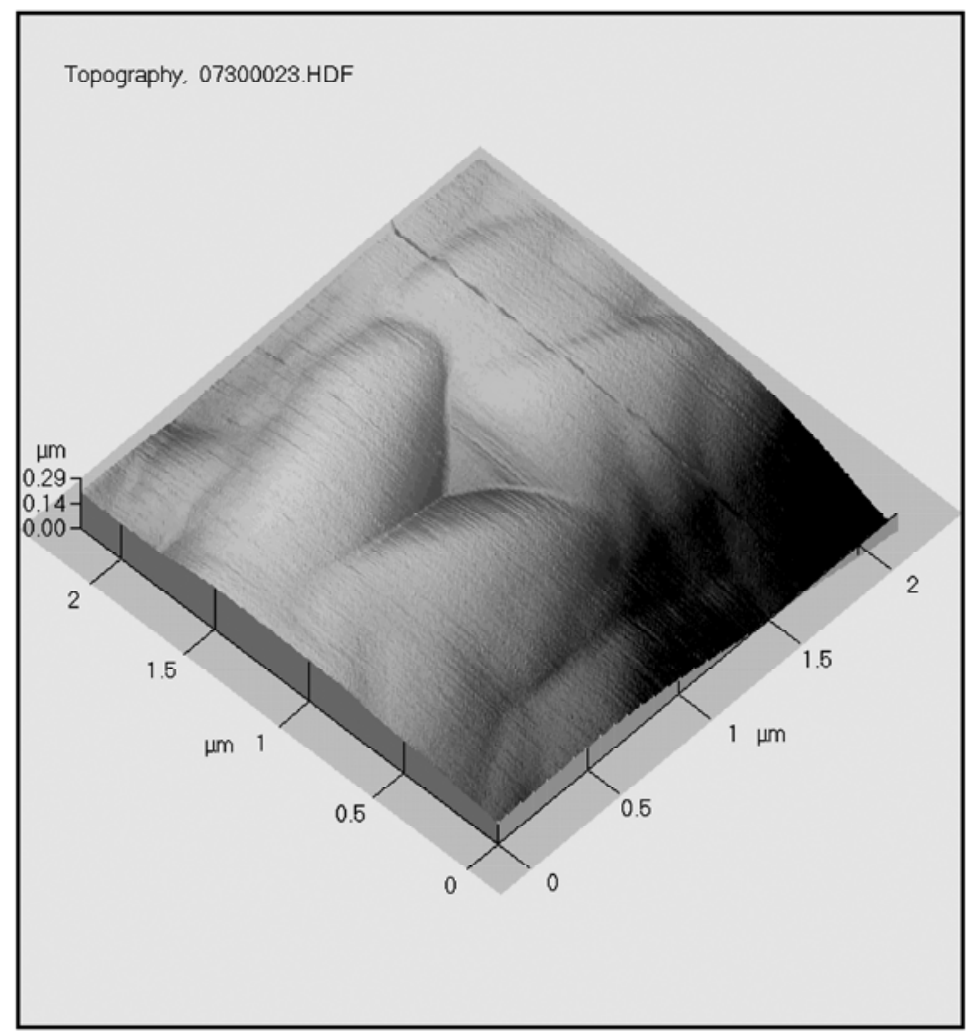

Figura 8: Vista 3D de una nanoindentacion sobre la capa $\mathrm{S} 2$ de Eucalyptus nitens realizada con indentador del tipo Berkovich. 


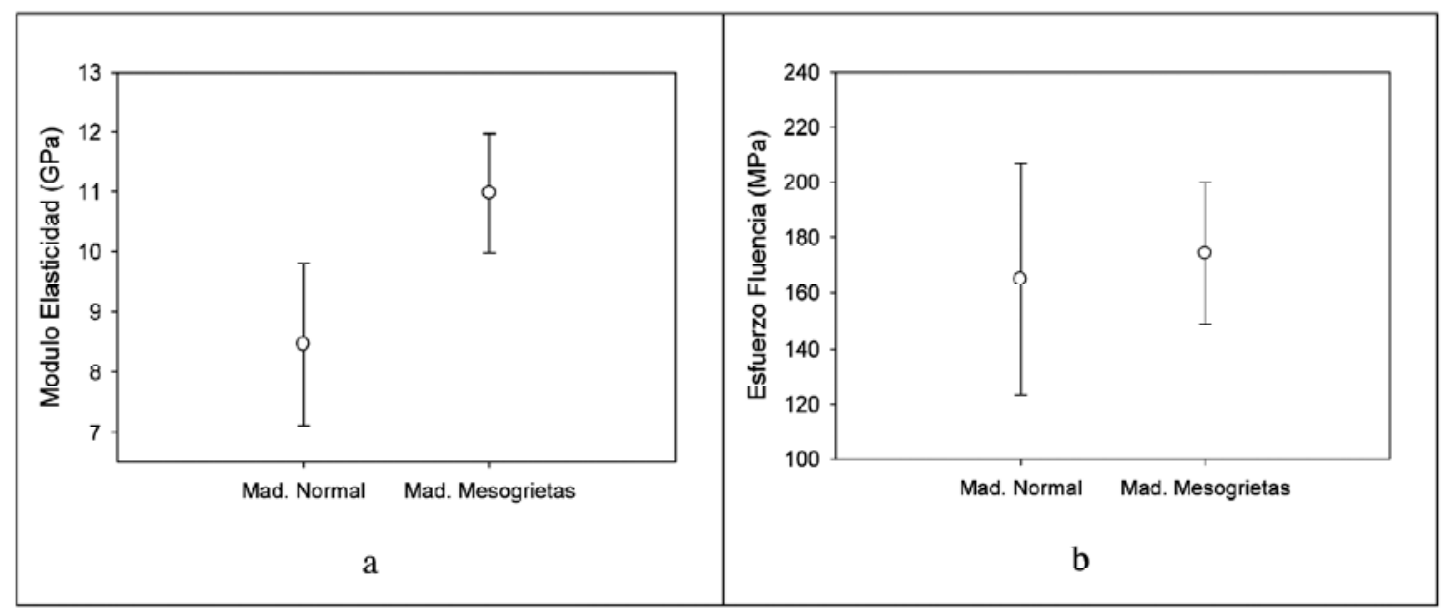

Figura 9: Intervalo de confianza (95\%) para la media del módulo de elasticidad (a) y esfuerzo de fluencia (b) de la capa S2 de células de Eucalyptus nitens con y sin mesogrietas.

\section{CONCLUSIONES}

El uso de nanoindentaciones en la capa S2 de células de Eucalyptus nitens combinadas con la evaluación de su ultraestructura, permitieron explorar en las causas de microgrietas y mesogrietas, frecuentes y visibles después de la corta del árbol y especialmente durante la transformación a productos de mayor valor. Hubo evidente colapso en muestras con mesogrietas. El colapso apareció principalmente alrededor del vaso debido a que allí se concentran las tensiones o esfuerzos. Una causa importante en la aparición de fallas en la estructura celular estaría relacionada con la frecuencia de vasos y sus características anatómicas. Por otro lado, se estableció que las microgrietas en Eucalyptus nitens se inician en la vecindad del vaso. Desde allí se propagan y generan mesogrietas y eventualmente rajaduras. En mesogrietas, la falla estuvo localizada fundamentalmente en la lámina media y pared S1. Otro factor significativo que contribuye a la aparición de fallas irrecuperables en el Eucalyptus nitens estaría asociado con la capacidad de las células de madera de absorber energía. Con nanoindentaciones se determinó que células de madera normal tienen la capacidad de absorber mayor energía de deformación antes de llegar éstas al colapso y eventualmente a la fractura.

\section{REFERENCIAS}

Alhasani, M.A. 1999. Growth stresses in Norway spruce. Report TVBK-1016, Division of structural engineering, Lund Institute of Technology, Lund, Sweden.

Bamber, R.K. 1978. Origins of growth stresses. Forpride Digest, 8(1): 75-96.

Gibson, L.; Ashby, M. 1997. Cellular solids, structure and properties. $2^{\text {nd }}$ edition, Cambridge University press.

Gindl, W.; Gupta, H.; Schoberl, T.; Lichtenegger, H.; Fratzl, P. 2004. Mechanical properties of spruce wood cell walls by nanoindentation. Appl. Phys. A 79: 2069-2073. 
Graig, R.R. 2000. Mechanics of materials. $2^{\text {nd }}$ edition. John Wiley \& Sons, Inc. Von Hoffman Press. ISBN 0-471-33176-7.

Leal, S.; Pereira, H.; Grabner, M.; Wimmer, R. 2003. Clonal and site variation of vessels in 7year-old Eucalyptus globulus. IAWA Journal. 24 (2): 185-195

Leitch, M.A. 2001. Vessel-element dimensions and frequency within the most current growth increment along the length of Eucalyptus globulus stems. Trees 15:353-357.

Sanford, R.J. 2003. Principles of fracture mechanics. Pearson Education Inc. Prentice Hall. ISBN 0-13-092992-1.

Smith, I.; Landis, E.; Gong, M. 2003. Fracture and Fatigue in Wood. John Wiley \& Sons Ltd. ISBN 0-471-48708-2

Ugural, A.C.; Fenster, S.K. 1995. Advanced strength and applied elasticity. $3^{\text {rd }}$ edition, Prentice Hall, Inc. ISBN 0-137589-X. 